\title{
XV. \\ Zur neuen Philo-Ausgabe.
}

I.

1) Am Schlufi des allgemeinen Teils der Prolegomena zur neuen Philo-Ausgabe ${ }^{1}$ ) schreibt L. Cohn in dem Abschnitt de ratione huius editionis (I p. LXXXIV) ${ }^{2}$ ):

1) veteris Testamenti locos quos attulit vel perstrinxit Philo quam accuratissime significavi LXX interpretum versionis editione novissima Cantabrigiensi (ed. Swete) usus, iuxta quam Lagardii editionem Geneseos (a. 1868) adhibui.

2 ) in scriptura locorum biblicorum constituenda codicum Philoneorum fidem ubique secutus sum, ac ne tum quidem ab ea discedendum esse iudicavi, ubi codices a versione graeca discrepant.

3) scripturam traditam ibi tantum e LXX virorum interpretatione correxi, ubi codices corruptos esse manifestum est.

4) sicubi codices Philonis in laudandis verbis biblicis inter se differunt, eam scripturam praeferendam esse duxi quae ad versionem graecam proxime accedat.

5) itaque non paucis locis codicum UF lectionem aspernatus ceterorum codicum memoriam secutus sum. nam ut ceteroquin manus correctrix in familia $U F$ grassata est (vide supra p. XL), ita et verba biblica corrector ille interdum immutavit, fortasse alia quadam veteris Testamenti versione usus.

Den Schlufsatz des ganzen Abschnittes über die Wichtigkeit der neuen Ausgabe für das Verhältnis von Philo und der griechischen Uebersetzung des Alten Testaments kann ich hier weglassen.

1) Philonis Alexandrini opera quae supersunt ediderunt Leopoldus Cohn et Paulus Wendland. Berolini, G. Reimer. Bis jetzt erschien Bd. I von Cohn 1896, Bd. 2 u. 3 von Wendland 1897. 1898. von mir.

$\left.{ }^{2}\right)$ Die Zählung und Hervorhebung im folgenden Druck stammt 
2) Noch ehe ich diese Grundsätze über die Behandlung der Bibelcitate, insbesondere über die Beurteilung der Handschriftenklasse UF und ihrer Citate kannte, war mir bei der Lektüre des Textes - denn von genauem Durcharbeiten desselben kann ich bei meiner beschränkten Zeit nicht reden aufgefallen, dak der Herausgeber den Text biblischer Stellen mehrfach anders beurteilte und herstellte, als ich es gethan hätte. Um mit dem Beispiel zu beginnen, bei dem mir das zuerst am meisten auffiel.

S. $53^{3}$ ) druckt Cohn einen auf Lev. 19, 23 anspielenden

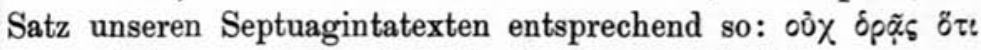

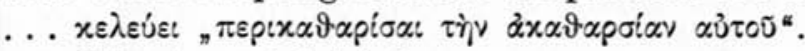

Sein Apparat belehrt uns:

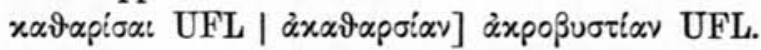

Letzteres Wort fiel mir auf, und so habe ich zu der Stelle die Ausgabe von Holmes (-Parsons) nachgeschlagen: - es ist mir unfaßilich, wie ein Herausgeber der Werke Philo's sich auf Swete und Lagarde beschränken mag, und dafs es bisher sogar zwei thaten, Cohn und Wendland, ist noch unbegreiflicher. Bei Holmes findet sich nun zwar zu dieser Stelle nicht viel, immerhin die Notiz, daf statt $\pi \varepsilon \rho\llcorner x \alpha \vartheta \alpha \rho \iota \varepsilon i \tau \varepsilon$ des über-

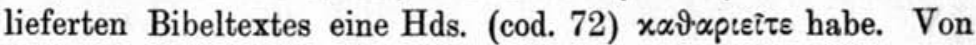
der Variante $\alpha x p \circ \beta u \sigma \tau i \alpha \nu$ für $\alpha x \alpha \vartheta \alpha p \sigma i \alpha \nu$ keine Spur. Um so mehr schien mir für Philo das Simplex des Verbums, das

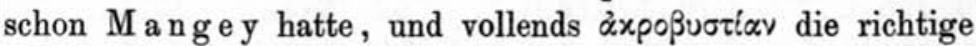
Lesart. Wenn man nemlich bedenkt, daßs im hebräischen Text ערלתו "Vorhaut" steht, und von Bä umen die Rede ist, deren Vorhaut d. h. ersten Früchte man nicht wegnehmen soll, so ist doch die Annahme das nächstliegende, das wörtlichere

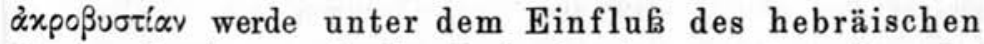
Textes für das umschreibende $\alpha x \alpha \vartheta \alpha p \sigma i \alpha \nu$ gesetzt worden sein, also: da wir es mit einem jüdischen Schriftsteller zu thun haben, die Handschriftengruppe UF habe uns das er-

$\left.{ }^{3}\right)$ Ich citiere nach den Seiten Mangey's, die in der neuen Ausgabe am Rand angeführt sind, um das Folgende auch denen zugänglich zu machen, welche die neue Ausgabe nicht haben. In derselben sind die biblischen Stellen in lat. Schrift im Context namhaft gemacht, also leicht zu finden; wo es irgend nötig ist, nenne ich Seiten und Zeilen der neuen Ausgabe.

Philologus LIX (N. F. XII), 2.Brought to you by | University qif Texas at El Paso 
halten, was Philo schrieb, in den andern sei es nach dem in der Kirche verbreiteten Septuaginta-Text korrigiert worden.

3) Eine andre Stelle, die mir auffiel, war der Eingang des dritten Buchs der Legum Allegoria (p. 87).

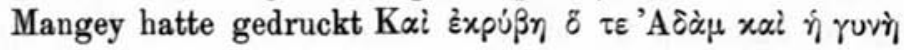

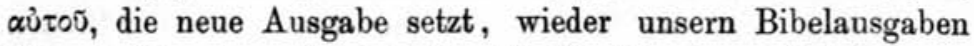

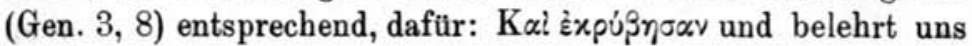
im Apparat:

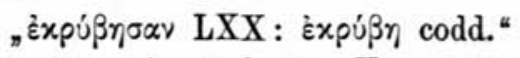

Für den von mir geplanten Kommentar zur Septuaginta (s. Prot. Real. Enc. ${ }^{3}$ 3, 19 Z. 35) hatte ich mir schon längst

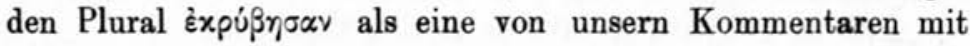
Stillschweigen übergangene Abweichung des griechischen Textes vom hebräischen notiert, und war um so mehr erfreut, jetzt in Philo d. h. in den Philohandschriften den Singular zu finden. Eigentlich hätte ich ihn, wenn die Philocitate bei Holmes vollständig gewesen wären, schon längst aus Holmes kennen sollen, da wie ich jetzt sehe schon Mangey ihn hatte; aber bei Holmes fehlt dies. Dagegen hätte Cohn bei ihm gefunden

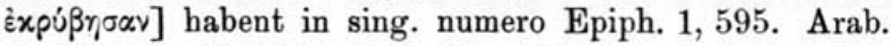

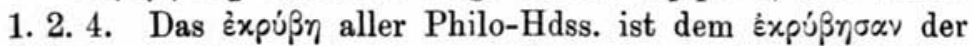
LXX gegenüber wieder eine Annäherung an den hebräischen Text, wie sich solche bei Philo massenhaft finden, z. B. gleich im nächsten Vers die Weglassung des Vokativs 'Aóá

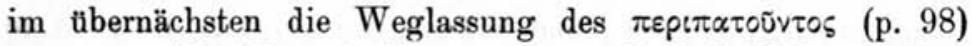
u. s. w. Allerdings heifst es nun auch bei Philo nachher

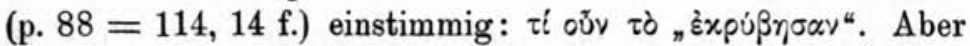
eben an dieser Stelle fährt Philo selbst im Singular fort, in-

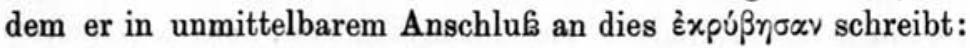

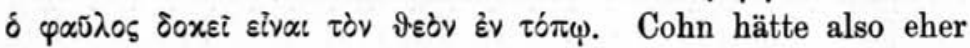
hier den Plural in den Singular verwandeln sollen, als umgekehrt vorher den Singular in den Plural, da wo die Stelle erstmals, und zwar im Zusammenhang, also genauer, citiert wird. Uebrigens ist es in solchen Fällen vielleicht geratener, es im Text bei der Lesart der Handschriften zu lassen und nur im Apparat auf die Verschiedenheit aufmerksam zu machen.

4) Aber nun noch eine dritte Stelle, der gegenüber das Verfahren der neuen Philo-Herausgeber ganz unbegreiflich 


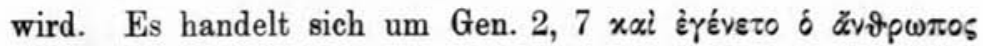

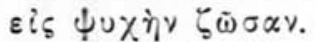

S. $49(=69,1)$, wo die Stelle von Philo erstmals citiert wird, druckt Cohn genau so wie in unsern Septuaginta-Ausgaben; der Apparat aber belehrt uns

$\zeta \tilde{\omega} \sigma \alpha \nu$ UFL Arm: $\omega_{\omega} \tilde{\zeta}_{\varsigma}$ MAP.

Nun hätte ich schon nach Bengels textkritischem Grundsatz: proclivi scriptioni praestat ardua, den man in der kürzeren, aber minder richtigen Form lectio difficilior placet anzuführen

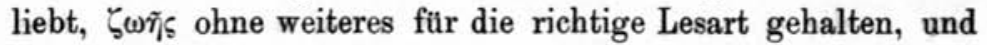
Cohn's Entscheidung wurde mir vollends unverständlich, als er einige Zeilen weiter wieder druckt (p. $50=69,11$ )

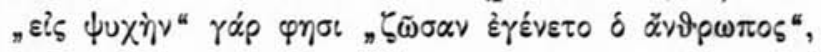

wo er im Apparat uns belehren muf

$$
\zeta \tilde{\omega} \sigma \alpha \nu] \zeta \omega \tilde{r}_{\varsigma} \text { codd. }
$$

und wo deshalb schon Mangey $\zeta_{\omega} \tilde{\zeta}$ gehabt hatte.

Die Stelle kommt aber bei Philo noch öfter vor. Zum drittenmal p. 119. Wieder hatte Mangey $\zeta \omega \tilde{\zeta}$, wieder setzt Cohn im Text $\zeta \tilde{\omega} \sigma \alpha v$, und belehrt uns im Apparat

$$
\zeta \tilde{\omega} \sigma \alpha v] \zeta \omega \tilde{\gamma} \text { codd. }
$$

Ein viertesmal citiert Philo den Vers p. 207. Cohn setzt

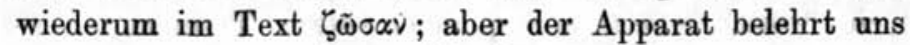

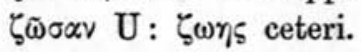

Aber nicht blok Cohn urteilt so, sondern auch Wendland.

Ich konnte bis jetzt nur den ersten Band genauer durchnehmen; die neue Ausgabe hat bis jetzt noch kein Citatenregister, aber wie ich aus Mangey's Register sehe, findet sich die Stelle ein fünftesmal 481 (= III, 14), und wieder heifst es im Text $\zeta \tilde{\omega} \sigma \alpha \nu$ und der Apparat belehrt uns

$$
\zeta \tilde{\omega \sigma \alpha \nu] ~ \zeta \omega \eta \varsigma ~ P a p . ~}
$$

Das heifat, der Papyrus, der einige Jahrhunderte älter ist als alle unsre Hdss., von dem Wendland selbst rühmt (III p. VIII) : Papyri fides etiam in locis S. S. afferendis agnoscitur u. s. w.) hat uns hier, und zwar hier er allein noch, das richtige enthalten, während endlich

beim letzten und sechstenmal, wo die Stelle vorkommt (S. $625=$ III, 212), das $\zeta \tilde{\omega} \sigma \alpha \nu$ des Bibeltextes, wie ich aus 
dem Stillschweigen des Apparats annehmen muf, in allen Zeugen zum Sieg durchgedrungen ist.

Die Stelle ist textkritisch im höchsten Maß3 lehrreich. Für das nach meiner, und gewif aller methodischen Textkritiker Ueberzeugung Richtige treten das erstemal vier auch sonst als gut erkannte Zeugen ein, gegen 3 ; beim 2 ten und drittenmal alle, beim vierten alle gegen einen, beim fünften noch einer, aber der beste, beim sechsten keiner mehr. Und trotzdem nehmen die neuen Herausgeber konsequent die unrichtige Lesart auf, während Mangey wenigstens zweimal bei seinen Handschriften geblieben war; und das thun sie zum Teil gegen ihre eigenen oben hervorgehobenen Grundsätze.

Daßs dieses $\zeta \omega \tilde{\eta} \varsigma$ auf Philo selbst und nicht etwa auf einen der andern Bibelübersetzer zurückgeht, können wir in diesem Fall um so sicherer behaupten, als wir zufällig zu diesem Vers, namentlich durch Philoponus (de opificio mundi, ed. Reichardt p. 273 f.) über die andern Uebersetzer, Aquila, Symmachus und Theodotion, genau unterrichtet sind. Und vornherein unwahrscheinlich ist ohnedies Cohns Annahme, dak ein Corrector in UF verba biblica interdum immutavit, man sieht nicht ein, wozu, fortasse alia quadam veteris Testamenti versione usus, man weifs nicht, welcher. (Unsere Handschriften sind ja alle ziemlich jung und gehen schließlich auf das Exemplar des Origenes in der Bibliothek von Cäsarea zurück).

Es ist leicht einzusehen, wie viel von der Entscheidung der hier aufgeworfenen Fragen abhängt. Bekomme ich recht, so ist gerade das das echte, was nach den neuen Herausgebern von einem Corrector herrührt. Und hat die Handschriftenklasse UF in den Bibelstellen vielfach das richtige erhalten, so erweckt dies ein Vorurteil auch für andere Stellen. Mit solchen allgemeinen Erwägungen ist natürlich nichts gethan; es handelt sich um eine Nachprüfung aller Bibelcitate Philo's und im Zusammenhang damit der übrigen Grundsätze, die der neuen Ausgabe zu Grunde liegen.

Wendland selbst hat unlängst so scharf auf Mängel aufmerksam gemacht, die seiner Meinung nach in dieser Hinsicht der neuen Origenes-Ausgabe der Berliner Akademie anhaften; 
um so mehr wird er geneigt sein, die vorstehenden Winke so freundlich aufzunehmen, wie sie gemeint sind. Da die neue Ausgabe in raschem Fortschreiten begriffen ist, will ich nicht warten, und veröffentliche dies, nachdem ich wenig mehr als 8 Tage auf Durcharbeiten des ersten Bandes habe verwenden können $\left.{ }^{4}\right)$.

II.

Der zweite Band der neuen Ausgabe wird eröffnet durch die nur in der einen Handschrift U erhaltene Schrift de posteritate Caini. Der Herausgeber (Wendland) hat in dieser Zeitschrift (57, 2 248-288) diejenigen Stellen besprochen, „die einer Erklärung oder Emendation bedürfen, oder die von andern nicht richtig behandelt worden sind", und hat die Gelegenheit benützt, um Philos Bedeutung für die Rekonstruktion der LXX anzudeuten. Auf letzteren Punkt will ich hier nicht mehr eingehen; aber einzelne Stellen bedürfen noch der $\mathrm{Be}-$ richtigung.

1) S. 236 citiert Philo $\mathrm{Nu}$. 13, 23 und etymologisiert die

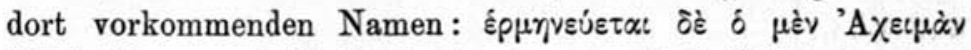

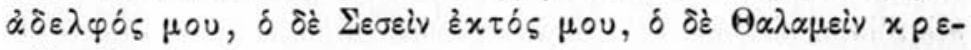
$\mu \dot{\alpha} \mu \varepsilon v \dot{\sigma}_{\varsigma} \tau$ is.

Es bedarf fürwahr nur sehr geringer Kenntnis des Hebräischen, um zu sehen, daßs $\Sigma \varepsilon \sigma \varepsilon \iota=$ zwar ЕКTOCMOY

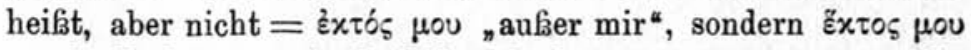
"mein Sechster". Auch $\Theta \alpha \lambda \alpha \mu \varepsilon i v$ ist genau genommen nicht

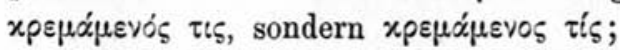

Diese Etymologie benutzt Philo sofort zu einer allego-

4) Rühmend möchte ich hervorheben, wie korrekt der erste Band gedruckt ist. Mir sind ganz wenige Versehen aufgestoßen: spros statt

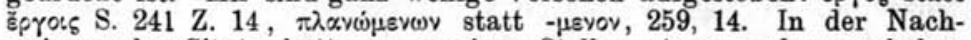
weisung der Citate hätte an wenigen Stellen etwas mehr geschehen

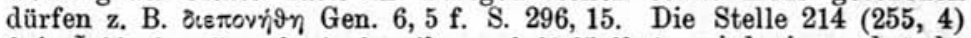

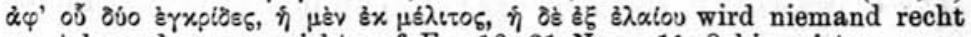
verstehen, den man nicht auf Ex. 16, 31 Num. 11, 8 hinweist.

Eine weitere Stelle, wo Philo sicher anders las als jetzt gedruckt

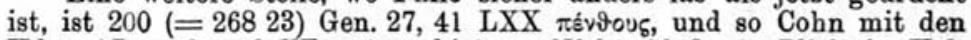
Hdss. AL, während UF $\pi \dot{\alpha} \vartheta$ ov bieten. Nicht bloß ein Blick in Holmes, sondern der Zusammenhang bei Philo beweist für $\pi \dot{\vartheta} \vartheta$ ợ.

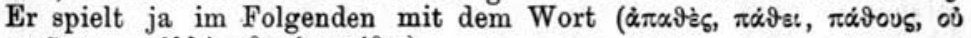

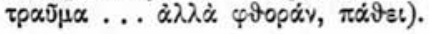




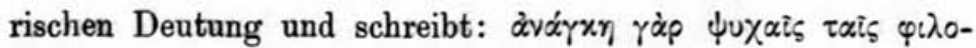

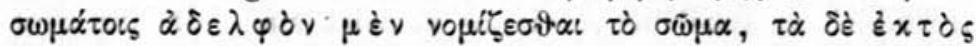

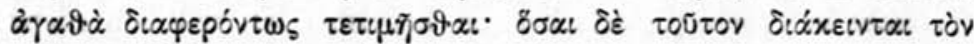

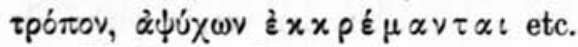

Nach dem Vorhergehenden ist anzunehmen, dafi auch hier nicht von „äufern Gütern“ die Rede sein kann ( $\grave{\alpha} \gamma \alpha \vartheta \alpha$ ), sondern daßs die Sechszahl hereinspielt. Und daßs dem so ist, beweist die Fortsetzung (S. 14 Z. 11), wo Philo aus-

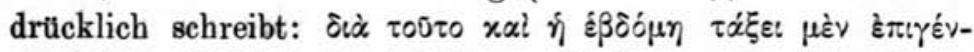

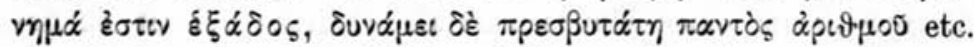

Es ist mir unbegreiflich, wie dies verkannt werden konnte. Ueber die Minderwertigkeit der Sechszahl gegenüber der Siebenzahl hat Philo schon $44(1,62)$ gehandelt $\left.^{5}\right)$.

2) Im gleichen Zusammenhang etymologisiert Philo über die hebräische Form des in der gleichen Bibelstelle vorkommenden ägyptischen Stadtnamens $\mathrm{Z}_{0}{ }^{\circ}$ an, $\mathrm{T} \alpha \dot{\alpha} \iota_{\varsigma}$, und schreibt

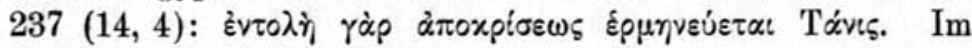
Apparat schreibt Wendland:

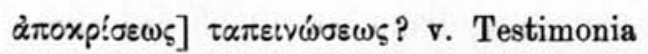

und verweist unter diesen auf die Onomastica sacra, wo Tanis

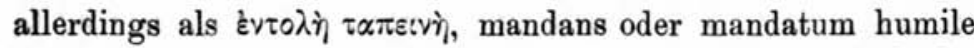
gedeutet ist. Aber im Hebräischen giebt es doch zwei gleichlautende Wurzeln 'ana, פנה, von denen die eine, weit häufigere, antworten, die andere gedrückt sein bedeutet. An die erste denkt Philo, an die zweite die Onomastica. Wozu also diese Verweisung?

3) $239(2,16)$ druckt Wendland in dem Citat Gen. 11, 29 $\varepsilon \bar{\lambda} \alpha \beta$ ○े, während die einzige Hds., auf welcher sein Text ruht, $\varepsilon \lambda \alpha \beta \varepsilon v$ hat. Genau so hat von LXX-Handschriften z. B. der Codex E, mit dem Philo oft genug stimmt; dazu noch einige Hdss. bei Holmes. Grabe hat sogar den Singular statt dem vom Alexandrinus gebotenen Plural als die richtige Lesart in seinen LXX-Text aufgenommen. Warum also den PhiloText ändern?

$\left.{ }^{5}\right)$ Eine weitere Stelle, die zeigt, daß 13, 21 statt der èx

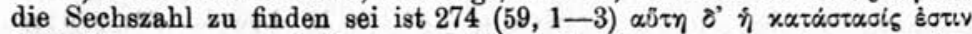

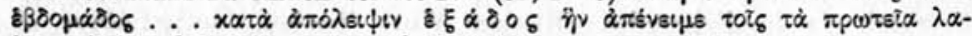

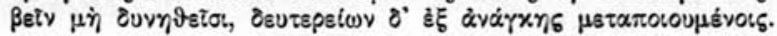




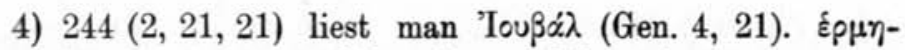

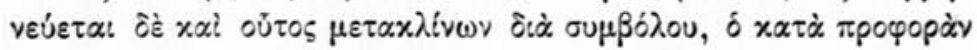
$\lambda$ bóros. Wer versteht bei solcher Interpunktion, daßs das heicen soll: „Jubal heißst „verändernd“, und bedeutet symbolisch

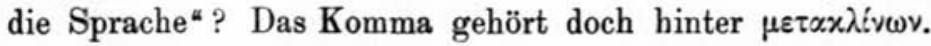

5) $249(2,27)$ ergänzt Wendland in dem Citat Gen. 4,25 $\langle\lambda \varepsilon ́ \gamma \circ u \sigma \alpha\rangle$ mit der Bemerkung ,ex LXX addidi". Wozu? Ein Blick in die LXX-Ausgabe von Holmes hätte gezeigt, dak

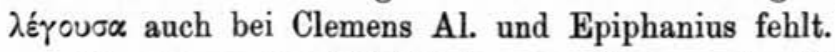

So viel zu den Bibelcitaten in dieser ersten Schrift des zweiten Bandes ${ }^{6}$ ).

III.

Höchst interessant ist gleich im Eingang der Schrift quod

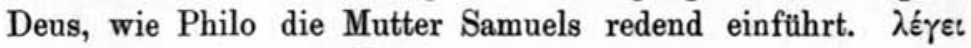

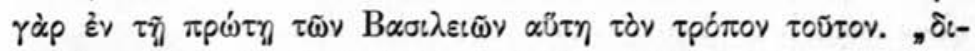

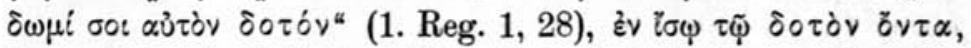

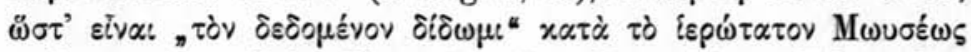

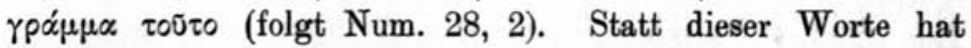

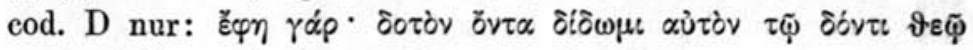

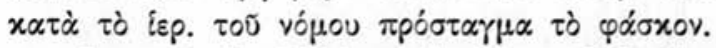

So nach der Textgestaltung von Wendland 57, Z. 15-18. Die Varianten der übrigen Handschriften sind unbedentend:

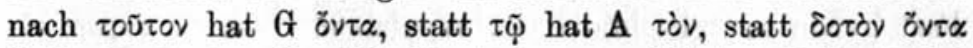
G $\delta \circ \tau \tilde{\omega} v \tilde{\omega}$ sic. Der Bibeltext der angeführten Stelle lautet

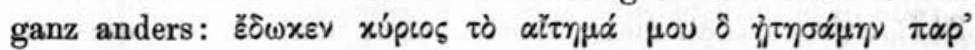

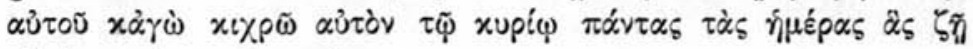

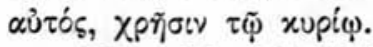

Was citiert Philo hier? Stellt man den Text so her, wie Wendland, dann scheint man annehmen zu müssen, dafs er eine zu seiner Zeit existierende griechische Uebersetzung wörtlich citiert; denn er kommentiert ja eigens einen Ausdruck des Citats, das Wort סotòv, von dem er sagt, es sei gleich-

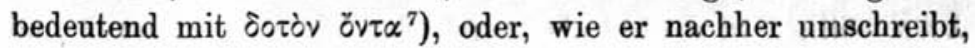

6) An Druckfehlern verbessere 37, 11 Deut. 32 statt 33 ; 54, 6 ov $\gamma$ -

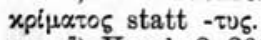

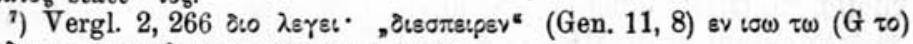

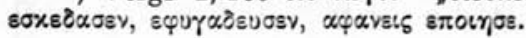




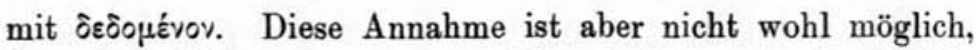
denn keine Uebersetzung von 1. Reg. 1, 28 konnte die direkte Anrede an Gott enthalten $\delta: \delta \omega \mu t$ oo ; das ist vielmehr For-

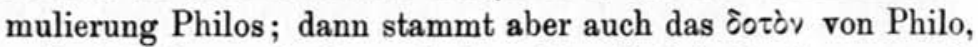

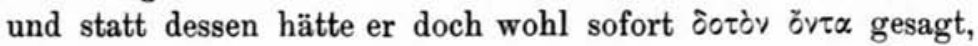
um seine Meinung deutlich auszudrücken. So legt sich die

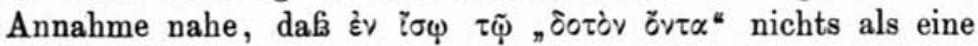
in den Text gekommene, in D entfernte Randbemerkung ist, wie $198(1,266)$, die so interessante, die uns belehrt, daf Ex. 7, 11 - die Stelle ist von Cohn nicht angeführt - $-\varphi \alpha \rho-$

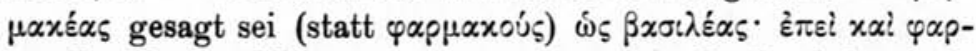

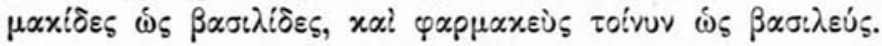

Will man dies nicht annehmen, dann mufs man entweder

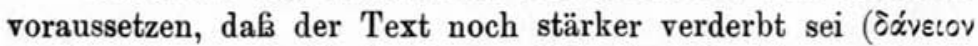

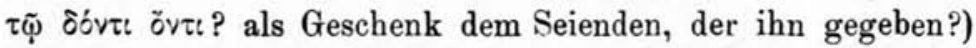
aber dazu liegt kein Grund vor; oder daß Philo aus irgend einem Grund sich selbst kommentiert.

2) Nun aber die Fortsetzung. Die von Philo citierte Bibelstelle Num. 28, 2 lautet wie in unsern Septuagintaaus-

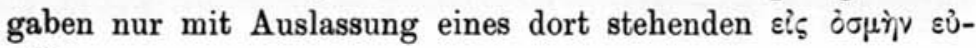
$\omega \delta i \alpha_{\varsigma}$ so:

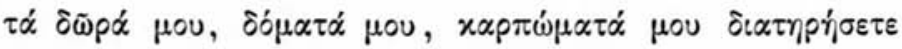

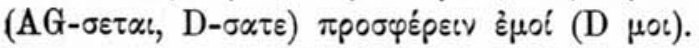

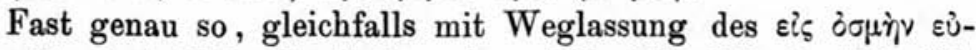

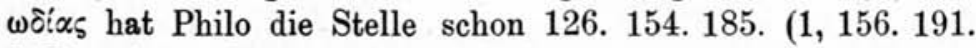
$247)$ citiert. Statt dieser Worte haben nun aber UF diesmal:

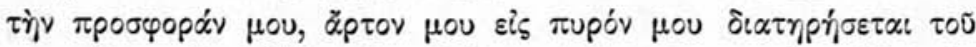

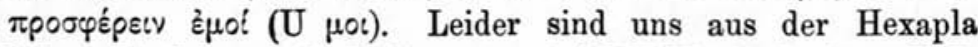
keine Varianten überliefert; in 2 LXX-Handschriften fehlt

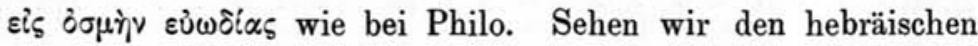
Text an, so entspricht demselben aufs genaueste die Lesart

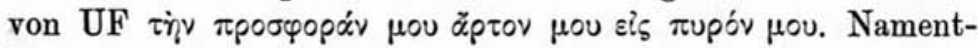
lich der letztere Ausdruck ist bemerkenswert. Er fehlt der

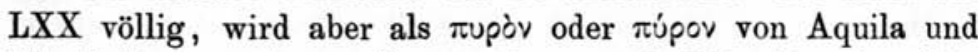
Theodotion, auch von Symmachus regelmäfig für das hier stehende gẹraucht (s. Field zu Lev. 2, 9). Für diese Stelle weif̧ ich einstweilen auch keine andere Erklärung als die Annahme von Wendland-Cohn, daf3 hier das Citat nach 
dem Grundtext korrigiert sei. Dabei ist aber auffallend, dafs dieser Korrektor, genau wie Philo, das zwischen אשמרו und משה stehende ריח unberücksichtigt läft; ob des Anthropomorphismus wegen? Oder sollte hier Philo selbst, wie er es unmittelbar vorher bei 1 . Reg. 1, 28 und auch sonst sicher gethan hat, vom griechischen Text abgewichen sein, und wiederum nur die Klasse UF uns das richtige bewahrt haben? Jedenfalls hat sie es (mit A) in der sofort zu besprechenden Stelle gethan.

275 (2, 59 f.) allegorisiert Philo, wie schon S. 260 (2, 39 f.) die Stelle Gen. 38, 9 und sagt schlieblich:

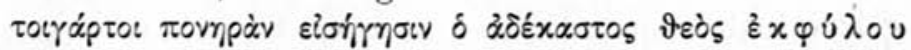

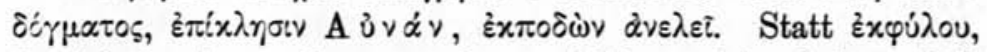
das ich in diesem Zusammenhang nicht verstehe, haben AUF

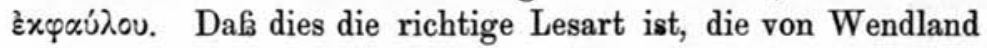
hätte in den Text gesetzt werden sollen, beweist die Etymo-

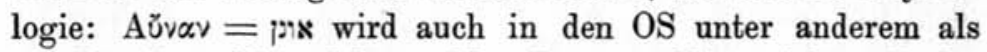

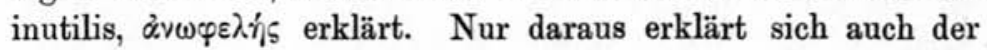

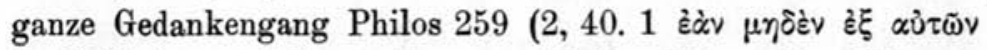

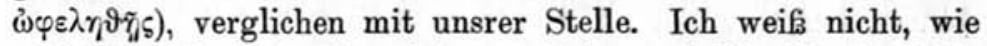
weit die hebräische Kenntnis von Cohn und Wendland reicht. $\mathrm{Da} 3$ in dem doppelt überlieferten Wort die Etymologie von Aủváv sich berge, mufite ein Bearbeiter Philos ohne eigene Kenntnis des Hebräischen merken; dann hätte er einen Hebraisten fragen müssen, und jeder hätte ihm die richtige $\mathrm{Be}-$ lehrung geben können; aus den Onomastica Lagarde's hätte man das Richtige sogar ohne Kenntnis des Hebräischen erschliefen können.

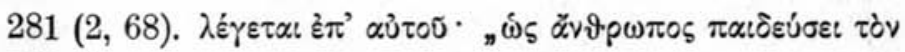

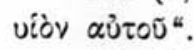

Wendland citiert dazu Deut. 1, 31, wo es heift $\omega_{\varsigma} \varepsilon l \tau$

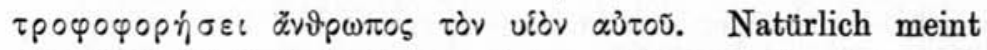

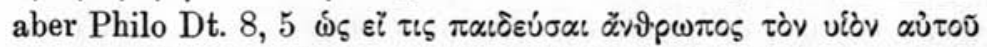

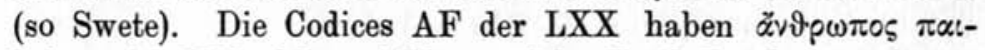

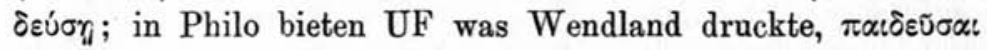
ceteri. Auch in Bd. 1, 243 wird Philo Dt. 8, 5, nicht 1, 31 im Auge haben. 3, 255 (= Mangey 1, 656), wo Mangey gleichfalls 1,31 citiert hat, hat Wendland jetzt die richtige Verweisung. 
Siehe Ryle, (Philo and Holy Scripture p. XXXVIII. XLIV. p. 246), der gleichfalls verkannt hat, dak es sich um ein Citat aus Dt 8, 5 handelt, und schrieb: Philo uses a more common

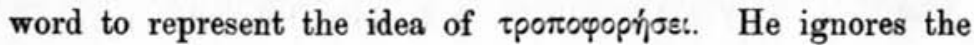
idea of carrying, which the Hebrew has and Aquila.

308 (2, 106). Das Citat aus Ex. 23, 20 druckt Wendland

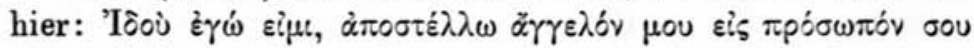

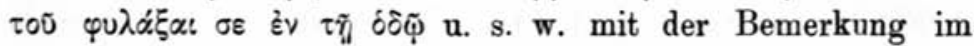
Apparat, dafis es nur UF, und $L$ am Rande, haben, die andern es weglassen, „fort. recte". Das mag sein; warum aber das Komma zwischen sì un ù $\dot{\pi} \pi \sigma \tau \dot{\varepsilon} \lambda \lambda \omega$ ? Es scheint den Herausgebern unbekannt, daf3 in gewissen Teilen der Septuaginta betontes ich, namentlich da, wo es hebräischem אנכ entspricht,

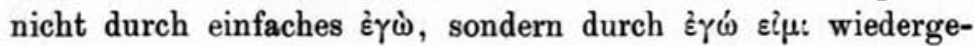
geben wird, an das sich das Verbum unmittelbar anschliefst. $\mathrm{Zu}$ den in der Koncordanz von Hatch-Redpath aufgeführten Beispielen füge aus Holmes-Parsons noch Ruth 2, 10.13. II. Reg.

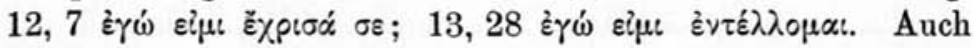

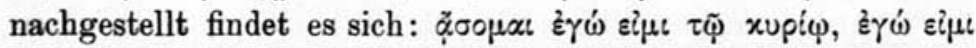
$\psi \alpha \lambda \tilde{\omega} \tau \tilde{\varphi}$ xupí $\varphi$, Jud. 5, 2. Es ist sehr lehrreich, diesen Sprachgebrauch hier zu finden. P. 463, wo die Stelle nochmals kommt,

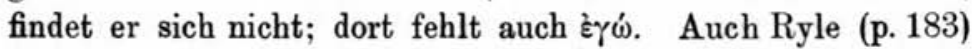

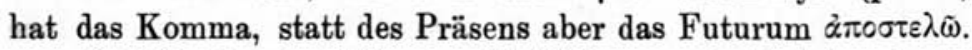

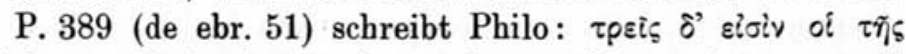

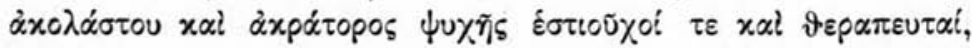

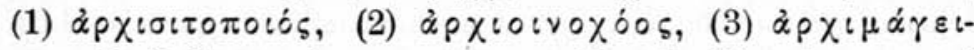

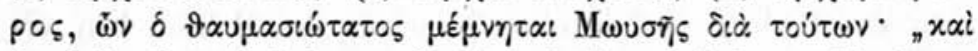

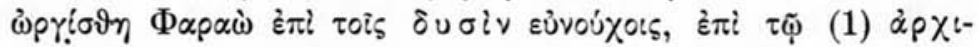

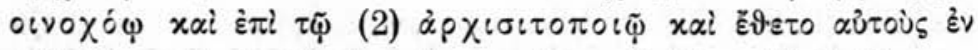

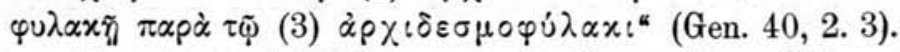

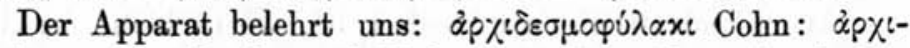

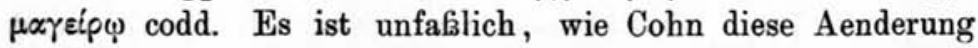
vorschlagen, wie Wendland sie annehmen konnte. Vollends

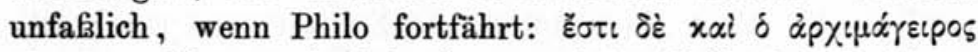

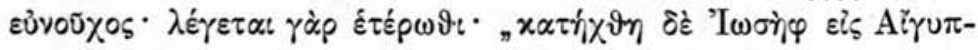

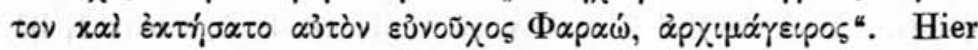
bewies doch der Context, daßs Philo in seiner Bibel $\alpha \rho \chi \psi \mu \alpha-$ $\gamma \varepsilon i p \varphi$ gelesen hatte. Und wenn man nun Holmes nachschlägt 
und da findet, daß 7 LXX-Handschriften samt dem Copten

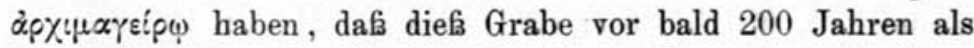

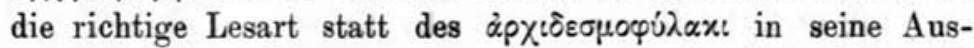
gabe des Alexandrinus einsetzte, wenn man beachtet, dafs auch

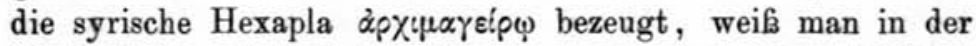
That nicht, mit welchem Namen man eine solche Textän-

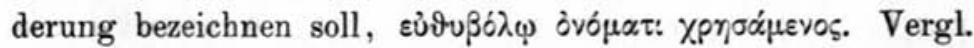
auch noch $390(212,14)$, wo die 3 wieder zusammengestellt

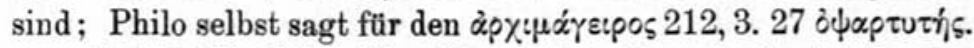

P. $411(2,237)$. Die Klage des Jeremia lautet in der

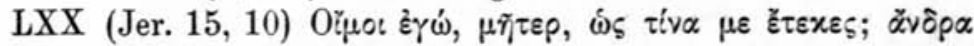

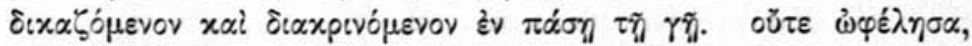

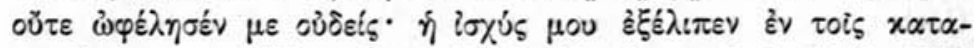

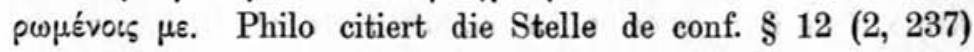
sich teilweise genau an das Hebräische anschliefend $\dot{\omega} \mu \tilde{\eta} \tau \varepsilon \rho$,

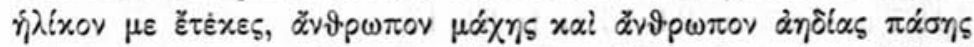

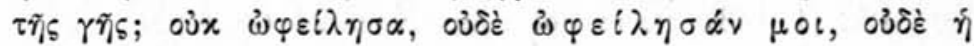

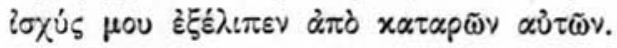

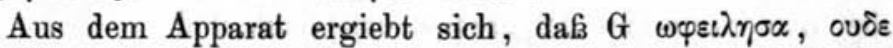

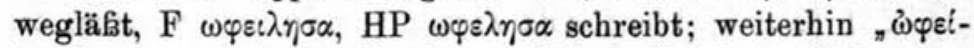

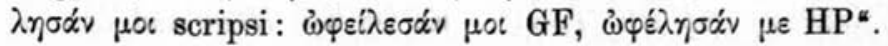

Die alten Ausgaben boten die Lesart von HP, die Wendland mit Unrecht verläßt. Es ist ja auf Grund des Hebräischen נשה fast selbstverständlich, daß $\omega \varphi \varepsilon i \lambda \eta{ }^{\prime} \sigma \alpha$ und $\omega \varphi \varepsilon i \lambda \eta \sigma \varepsilon$ die richtige LXX-Lesart ist, und so habe ich sie schon seit ge-

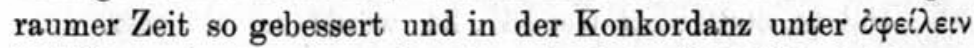

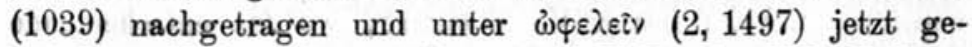
strichen. Nichts destoweniger wird Philo den Schreibfehler $\omega \varphi \hat{\varepsilon} \lambda \eta \sigma \alpha$ und $\dot{\omega} \varphi \hat{\varepsilon} \lambda \eta j \sigma \alpha \nu$ schon vorgefunden haben; das geht aus der Umschreibung hervor, die er S. $239 \mathrm{Z}$. 4 ff., wo er

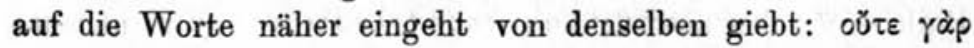

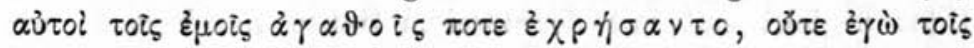

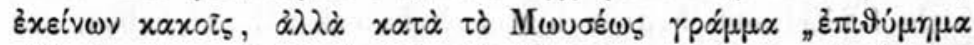

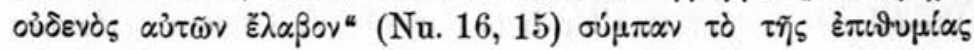

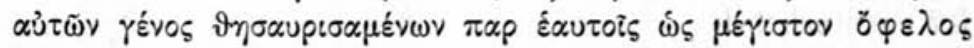

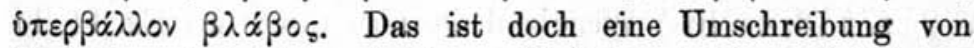
$\dot{\omega} \varphi \varepsilon \lambda \varepsilon i v$ und nicht von $\delta \varphi \varepsilon i \lambda \varepsilon เ v$. Statt $\delta \pi \varepsilon \rho \beta \dot{\alpha} \lambda \lambda$ ov hat $\mathrm{F} \delta \pi \varepsilon \rho-$ $\beta \alpha ́ \lambda$ ov, es ist wohl ó $\pi \dot{\varepsilon} \lambda \alpha \beta$ ov zu lesen. Nach dem Wort des 
Moses „०ưx ع̌ $\lambda \alpha \beta$ ov" achtete ich alles was sie als Nutzen an-

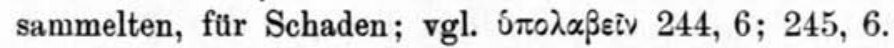

$462(2,301)$ Eine weitere Stelle, die durch einfache Interpunktionsänderung herzustellen ist. Philo schrieb N $\alpha \delta \grave{\alpha} \beta \delta$

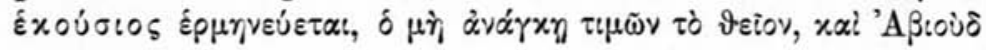

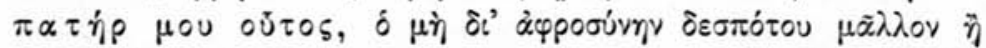

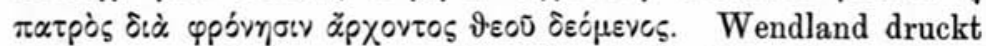
$\pi \alpha \tau \dot{n} \rho$ אביהוא ist $=$ mein Vater ist dieser) und den Parallelismus.

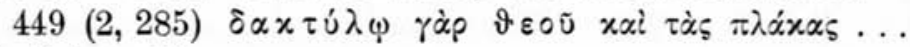

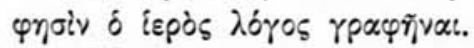

Wendland citiert dazu Ex. 32, 16, wo vom Finger Gottes, auf dem nach dem Zusammenhang der Nachdruck liegt, keine Rede ist; gemeint ist natürlich Ex. 31, $16 \pi \lambda \alpha \times \alpha \varsigma \lambda_{\text {t- }}$

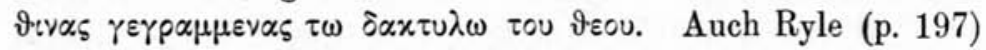
hat das Citat zu 32, 16 statt 31, 16.

$132(1,163)$ citiert Cohn aus Num. 21, (27-)30

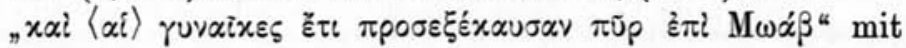
der Bemerkung im Apparat $\alpha i$ om. codd. und

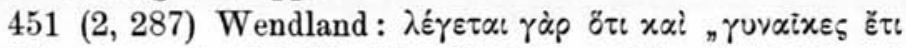

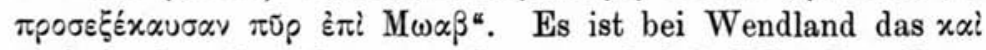
noch in das Citat hereinzunehmen und bei Cohn das $\alpha i$ um so weniger beizufügen als es auch in einzelnen Septuaginta-

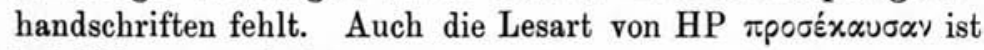
bei Holmes zu finden.

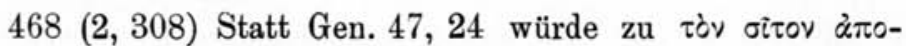

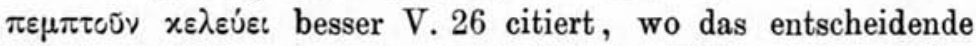
Wort wirklich vorkommt. Auch Ryle (p. 135) hat das minder richtige Citat von Mangey übernommen.

474 (Bd. 3, S. 2. 3.) Wendland citiert hiezu Gen. 26, 3-5;

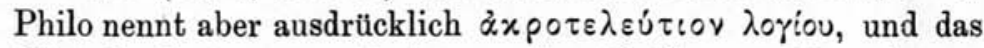
Citat beginnt erst mit der zweiten Hälfte von Vers 4 , wo viele LXX-Handschriften hinter $\delta \omega \sigma \omega$ das $\sigma o \grave{i} x \alpha \grave{\imath}$ haben, das Wendland aus Swete allerdings nur für V. 3 kennt. Holmes ist unentbehrlich.

$474(3,4)$ liest Wendland in dem Citat aus Ex. 14, 14 mit den Handschriften $\sigma \succ \gamma \eta \dot{\sigma} \sigma \varepsilon \tau \varepsilon$, während der sonst vorzuziehende Papyrus „ $\sigma \omega \pi \dot{\eta} \sigma \varepsilon \sigma \vartheta \varepsilon$ " bietet. Jedenfalls wird $\sigma \cdot \gamma \dot{\eta} \sigma \varepsilon \sigma \vartheta \varepsilon$ zu 
lesen sein; es läßst sich sogar fragen, ob der Papyrus nicht so habe, da in der vorangehenden Zeile Scheil nach Wendland ein $\gamma$ zu $p \omega$ verlesen konnte. Die phototypische Tafel ist mir nicht zur Hand.

$475(3,5)$ druckt Wendland in dem Citat aus Ex. 20, 19 $\mu \eta \dot{\eta} \pi 0 \tau \varepsilon \alpha \pi \circ \vartheta \dot{\alpha} v \omega \mu \varepsilon v$. Aber der Apparat belehrt, daf $\pi \circ \tau \varepsilon$ im Papyrus fehlt, und ein Blick in Holmes hätte gezeigt "sic (nemlich $\mu \eta \pi о \tau \varepsilon$ ) et Philo 1, 475 sed $\mu$ ì 1, 238. 642“. 1, 238 muf ein Druckfehler sein für 1,253 (=W 2,32), wo nach dem Schweigen des Apparats alle Hdss. nur $\mu \grave{\eta}$ haben; 642 ist $=\mathrm{W} 3,235$, wo $\mathrm{W}$ jetzt allerdings auch $\mu \dot{\eta} \pi \circ \tau \varepsilon$ druckt, laut Apparat aber GFHP $\pi \circ \tau \varepsilon$ weglassen; also ist es doch wahrhaftig geratener an allen Stellen nur $\mu \grave{r}$ zu setzen.

$476(3,7) \mathrm{Je}$ seltener Citate Philos aus den Propheten sind, um so weniger hätte zu $\sigma \nu \mu \circ \quad \gamma \lambda \omega \sigma \sigma \alpha \nu \pi \alpha i \delta \varepsilon \alpha_{\varsigma} \varepsilon \delta \omega x \alpha \varsigma$

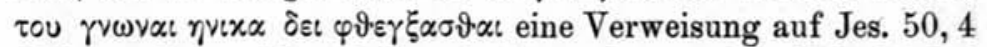
fehlen sollen. Man sieht wie Lagarde, an den Heinrici bei Besprechung des ersten Bandes in der ThLz 1897. 8. 214 erinnert, hinsichtlich der Indices recht hatte. Das Citat fehlt auch bei Ryle, p. 297.

$495(3,37)$ Statt $\alpha \check{\delta} เ x \alpha$ vermutet W. in dem Citat aus

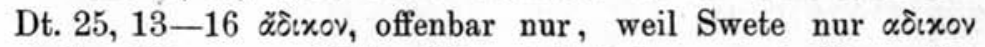
giebt. Eine ganze Reihe Hdss. bei Holmes, auch Clem. Al., haben den Plural; $\alpha \delta(x i \alpha \nu$ in der Philohandschrift $G$ ist durch den Zusammenhang veranlafit (s. Z. 12. 20).

$506(3,54)$ schreibt Philo von den schenkelbesitzenden,

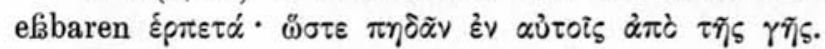

W. bemerkt im Apparat „ $\dot{\alpha} \pi \dot{\jmath}]$ ह̇ $\pi \grave{\imath}$ conicio “, offenbar, weil die gedruckten Septuagintatexte so haben. Aber Philo sagt

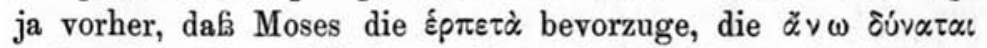

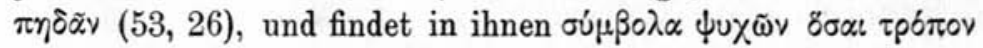

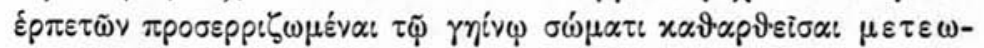

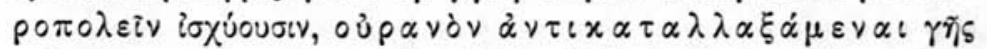
$x \alpha i \quad \varphi \vartheta \nabla \rho \tilde{\alpha} \varsigma \alpha \vartheta \alpha v \alpha \sigma i \alpha \nu$. Also ist das $\alpha \pi \dot{b}$ durch den Zusammenhang gesichert, und wird zudem vom Septuagintacodex 75 geboten, der oft genug mit Philo übereinstimmt.

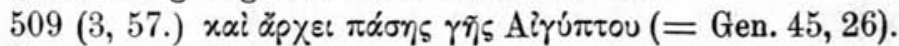

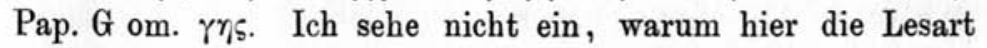


des Papyrus nicht mafgebend sein soll; auch in einzelnen Septuagintahandschriften fehlt $\gamma \tilde{\eta}$ s.

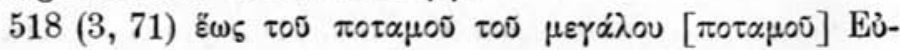

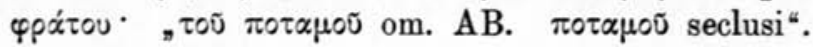

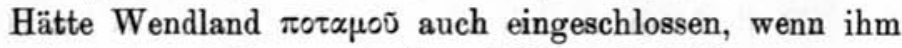
bekannt gewesen wäre, was schon Holmes bietet?

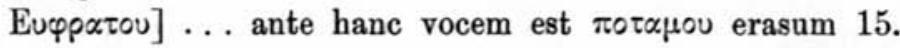

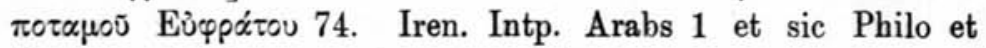
Aug. semel uterque, sed neuter ubique.

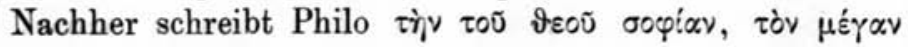

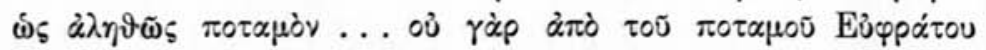

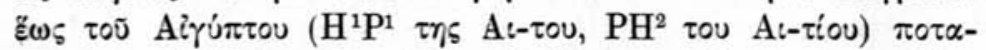

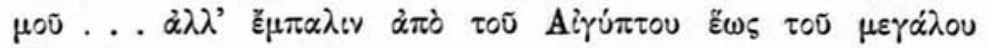

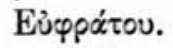

Man wird den Text lassen dürfen, aber vielleicht das Komma nach dem ersten $\pi 0 \tau \alpha \mu \circ$ setzen müssen, nicht nach $\mu \varepsilon \gamma \alpha \dot{\lambda}$ ou. $537(3,98$.) Ganz falsch verstanden hat Wendland den Passus

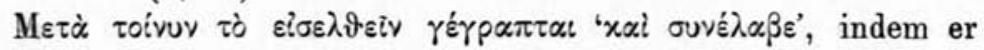
dies auf die vorher erwähnte Geschichte von Juda und Thamar bezieht Gen. 38, 14-18, während Philo mit diesen Worten zur Geschichte von Abraham und Hagar zurückkehrt (Gen. 16, 4

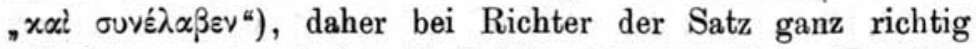
mit einem großen Anfangsbuchstaben begonnen ist. Um allen

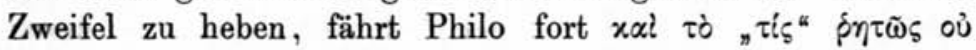
$\mu \varepsilon \mu \dot{\gamma} v u \tau \alpha t$ und weist damit schon hin auf seine seltsame Deutung

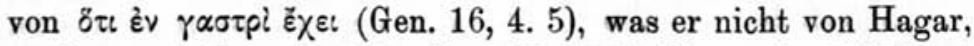
sondern von Sara deutet. Gen. 38, 18 heikt es im Bibeltext auch gar nicht $\sigma u v \varepsilon \hat{\lambda} \alpha \beta \varepsilon$, sondern żv $\gamma \alpha \sigma \tau \rho i$ ž $\lambda \alpha \beta \varepsilon$. Ebenso zeigt das 'èv $\gamma \alpha \sigma \tau \rho$ ' Gen. 16 ist.

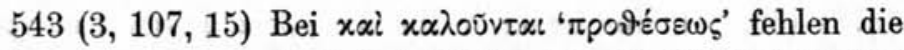
Anführungszeichen und die Bibelstellen Ex. 39, 18 (36). 40, 23. Es sind die einzigen, wo im Pentateuch die "Schaubrote" so genannt werden; an erster Stelle nicht in allen Handschriften

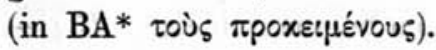


$\mathrm{Schlufb}$ emerkung.

Die im Vorhergehenden besprochenen Stellen geben keine Lösung der im Eingang berührten Hauptfrage, werden aber als Besserungen einzelner, zum Teil althergebrachter Versehen nicht umsonst sein. Was für ein interessantes textgeschichtliches Problem in Philo vorliegt, soll zum Schluf nur noch an einem seiner wenigen Psalmencitate klargestellt werden :

285 (2, 74, Quod Deus c. 18) citiert Philo Ps. 61 (62), 12. $\alpha \pi \alpha \xi$ xúpเos $\varepsilon \lambda \alpha \dot{\lambda} \lambda \eta\rceil \varepsilon$,

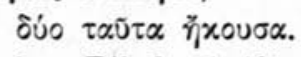

In unsern griechischen Bibeln heift es ohne alle Varianten der Handschriften:

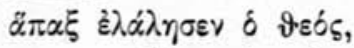

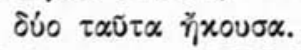

Die oben hervorgehobenen zwei Philohandschriften UF bieten statt dessen :

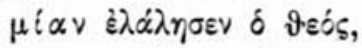

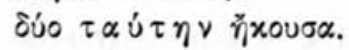

So hat keine der sonst bekannten alten Uebersetzungen (Aquila, Symmachus, Theodotion); dies entspricht aber genau dem hebräischen Grundtext: $\mu \dot{i} \alpha \nu=\mathrm{n} \mathrm{n}, \tau \alpha u ́ \tau \eta \nu=\eta=\uparrow($ Ps. 132, 12) $=$ ז Ps. 118, 23. Dieser Hebraismus, das Femininum statt des Neutrums zu gebrauchen, findet sich im griechischen Neuen Testament nur Mt 21, $42=$ Mc 12, 11 in dem Citat aus der letztgenannten Psalmstelle (vgl. Blass, Grammatik des Neutestamentlichen Griechisch $\S 32,2)$. Wie kommt ein so sel-

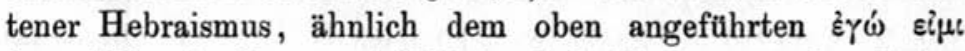
$\alpha \pi \tau \sigma \tau \varepsilon \hat{\varepsilon} \lambda \omega$, in diese zwei Philohandschriften, wenn sie mit den andern auf das Exemplar in der Bibliothek des Origenes zurückgehen? Es liegt hier ein text-, sprach- und kultur-geschichliches Problem vor, das noch genauer Untersuchung bedarf.

Maulbronn.

Eb. Nestle. 